\title{
Sumudu transform of Dixon elliptic functions with non-zero modulus as Quasi C fractions and its Hankel determinants
}

\author{
Adem Kilicman ${ }^{1, \star}$ and Rathinavel Silambarasan ${ }^{2}$
}

\author{
${ }^{1}$ Department of Mathematics, Faculty of Science, Universiti Putra Malaysia, 43400 UPM Serdang, Selangor, Malaysia. \\ Email : akilic@upm.edu.my \\ ${ }^{2}$ Department of Information Technology, School of Information Technology and Engineering, Vellore Institute of \\ Technology, Vellore, 632014, Tamilnadu, India. Email : silambu_vel@yahoo.co.in \\ * Corresponding Author.
}

\begin{abstract}
Sumudu transform of the Dixon elliptic function with non zero modulus $\alpha \neq 0$ for arbitrary powers $\operatorname{sm}^{N}(x, \alpha) ; N \geq$ $1, \operatorname{sm}^{N}(x, \alpha) c m(x, \alpha) ; N \geq 0$ and $\operatorname{sm}^{N}(x, \alpha) c m^{2}(x, \alpha) ; N \geq 0$ is given by product of Quasi C fractions. Next by assuming denominators of Quasi $C$ fraction to 1 and hence applying Heliermann correspondance relating formal power series (Maclaurin series of Dixon elliptic functions) and regular $\mathrm{C}$ fraction, Hankel determinants are calculated and showed by taking $\alpha=0$ gives the Hankel determinants of regular $\mathrm{C}$ fraction. The derived results were back tracked to the Laplace transform of $\operatorname{sm}(x, \alpha), \operatorname{cm}(x, \alpha)$ and $\operatorname{sm}(x, \alpha) \operatorname{cm}(x, \alpha)$.
\end{abstract}

Keywords : Dixon elliptic functions, non-zero modulus, Sumudu transform, Hankel determinants, Continued fractions, Quasi C fractions.

Mathematics subject classification : 33E05, 44A10, 11A55, 11C20.

\section{Introduction}

To determine the coeffecients in the Maclaurin series of Jacobi elliptic functions, continued fractions and the Heilermann correspondence the relation employing Formal Power Series (FPS) and its continued fraction to calculate Hankel determinants are used in [2], also determinants of Bernoulli numbers were calculated from the correspondence in [2]. By using continued fraction and Fourier series expansions of Jacobi elliptic functions in [13] obtained orthogonal polynomials which are related to each other through multiplication formulas of Jacobi elliptic functions in [13]. Laplace transform of Jacobi elliptic functions expanded as continued fractions and shown their coeffecients are orthogonal polynomials and derived dual Hahn polynomials in [19]. Fourier series and continued fractions expansions of ratis of Jacobi elliptic functions and their Hankel determinants are given in [25] from which different ways of representing sum of square numbers derived in determinant forms in [25]. Laplace transform of bimodular Jacobi elliptic functions expanded as continued fractions in [14] and by modular transformation results were back tracked to unimodular Jacobi elliptic functions in [14].

A. C. Dixon studied the cubic curve $x^{3}+y^{3}-3 \alpha x y=1 ; \alpha \neq-1$ for the orthogonal polynomials, where the curve has double period in [16] which then give raise to two set of elliptic functions $\operatorname{sm}(x, \alpha)$ and $\operatorname{cm}(x, \alpha)$ now known as Dixon Elliptic Functions (DEF). The examples, its relation to hypergeometric series, modular transformation and formulae for their ratio given in [17]. When $\alpha=0$ in the above cubic curve, their series expansions and transformations studied in [18]. DEF were used in the study of conformal mapping and geographical structure of world maps in [1] addition and multiplication formulae for DEF are derived in [1]. Laplace transform applied for DEF for both the cases of $\alpha=0$ and $\alpha \neq 0$ to expand as set of continued fractions in [14]. The above cubis curve and its relation to Fermat curve is studied for the Urn representation and combinatorics in [15]. Number theory related results followed by [25] for factorial of numbers using DEF given in [4]. DEF relation to trefoil curves and relation to Weierstrass and its derivative functions shown in [23]. 
Fractional heat equations are solved using Sumudu transform in [3]. Sumudu transform embedded in decomposition method in [27] and in homotopy perturbation method to solve Klein-Gordon equations in [26]. Fractional Maxwell's equations solved with Sumudu transform in [28] and some differential equations with Sumudu transform in [29]. Fractional gas dynamics differential equations using Sumudu transform is solved in [5]. Sumudu transform definition for trigonometric functions and its infinite series expansions proved with examples comprising tables and properties in [6]. Maxwell's coupled equations solved with Sumudu transform for magnetic field solutions in TEMP waves given in [7]. Without using any of decomposition, perturbation (or) analysis techniques Sumudu transform of functions calculated by differentiating the function in [8]. Symbolic C++ program for Sumudu transform given in [8]. Sumudu transform applied for bimodular Jacobi elliptic functions [14] for arbitrary powers in [9] as associated continued fraction and their Hankel determinants. Applying modular transformation, Sumudu transform of $\tan (x)$ and $\sec (x)$ derived in [9]. Sumudu transform of $f(x)$ defined in the set $A=\left\{f(x)\left|\exists M, \tau_{1}, \tau_{2}>0,\right| f(x) \mid<M e^{\frac{|x|}{\tau_{j}}}\right.$, if $\left.x \in(-1)^{j} \times[0, \infty)\right\}$ given by integral equation.

$$
\mathbb{S}[f(x)](u) \stackrel{\text { def }}{=} F(u) \stackrel{\text { def }}{=} \int_{0}^{\infty} e^{-x} f(u x) d x=\frac{1}{u} \int_{0}^{\infty} e^{-\frac{1}{u}} f(x) d x ; u \in\left(-\tau_{1}, \tau_{2}\right) .
$$

In this work Sumudu transform applied for DEF of arbitrary powers $\operatorname{sm}^{N}(x, \alpha) ; N \geq 1, \operatorname{sm}^{N}(x, \alpha) c m(x, \alpha) ; N \geq 0$ and $\operatorname{sm}^{N}(x, \alpha) \mathrm{cm}^{2}(x, \alpha) ; N \geq 0$ and expanded as Quasi C Fractions (QCF). Using the numerator coeffecients of QCF, Hankel determinants are calculated by the correspondence connecting FPS and Regular C fractions through Sumudu transform.

\section{Preliminaries}

Cubic curve $x^{3}+y^{3}-3 \alpha x y=1 ;(\alpha \neq-1)$ studied for its orthogonal polynomials in [16] derived the two set of elliptic functions namely $\operatorname{sm}(x, \alpha)$ and $\operatorname{cm}(x, \alpha)$ which are having double period. Derivative of DEF (equations (1) and (3), page 171, [16] and equations (1.18) and (1.19), page 9, [14]) takes the following,

$$
\frac{d}{d x} \operatorname{sm}(x, \alpha)=c m^{2}(x, \alpha)-\alpha \operatorname{sm}(x, \alpha) \text { and } \frac{d}{d x} \operatorname{cm}(x, \alpha)=-\operatorname{sm}^{2}(x, \alpha)+\alpha c m(x, \alpha) .
$$

and have (equation (1.21), page 10, [14]),

$$
\operatorname{sm}(0, \alpha)=0 \text { and } \operatorname{cm}(0, \alpha)=1
$$

these functions satisfies the cubic curve mentioned above what is called Pythagorean theorem (equation (2), page $171,[16]$ and equation (1.22), page 10, [14]).

$$
\operatorname{sm}^{3}(x, \alpha)+c m^{3}(x, \alpha)-3 \alpha \operatorname{sm}(x, \alpha) c m(x, \alpha)=1 .
$$

Continued fraction notation is followed from (equation (2.1.4b), page 18, [20] and (equation (1.2.5'), page 8, [22]).

$$
\prod_{n=1}^{\infty} \frac{a_{n}}{b_{n}} \stackrel{\text { def }}{=} \frac{a_{1}}{b_{1}+\frac{a_{2}}{b_{2}+}} \frac{a_{3}}{b_{3}+\cdots} \stackrel{\text { def }}{=} \frac{a_{1}}{b_{1}+\frac{a_{2}}{b_{2}+\frac{a_{3}}{b_{3}+\frac{a_{4}}{b_{4}+\ddots}}} .}
$$

Definition 1. Let $a=\left\{a_{n}\right\}, b=\left\{b_{n}\right\}$ and $u$ is an indeterminate, then the continued fraction of following form is called C-fraction (equation (7.1.1), page 221, [20]), [30] and (equation (54.2), page 208, [31]).

$$
1+\prod_{n=1}^{\infty} \frac{a_{n} u^{\beta(n)}}{1}
$$


When the sequence $\beta(n)$ is constant then C-fraction is called Regular C fraction. And QCF has the following form.

$$
\frac{a_{0}}{b_{0}(u)+} \coprod_{n=1}^{\infty} \frac{a_{n} u}{b_{n}(u)}
$$

Sometimes the coeffecient $a_{n}=a_{n}(u)$ thus the coeffecients are functions of $u$ which can be seen in the main results of this work.

Definition 2. Let $c=\left\{c_{v}\right\}_{v=1}^{\infty}$ be a sequence in $\mathbb{C}$. Then the following $m \times m$ matrices are defined [14,20,25], whose determinants are denoted by respective $H_{m}^{(n)}$ and $\chi_{m}$.

$$
\begin{gathered}
H_{m}^{(n) \stackrel{\text { def }}{=}} H_{m}^{(n)}\left(c_{v}\right) \stackrel{\text { def }}{=} \operatorname{det}\left(\begin{array}{ccccc}
c_{n} & c_{n+1} & \cdots & c_{m+n-2} & c_{n+m-1} \\
c_{n+1} & c_{n+2} & \cdots & c_{m+n-1} & c_{m+n} \\
\vdots & \vdots & \ddots & \vdots & \vdots \\
c_{m+n-1} & c_{m+n} & \cdots & c_{2 m+n-3} & c_{2 m+n-2}
\end{array}\right) . \\
\chi_{m} \stackrel{\text { def }}{=} \chi_{m}\left(c_{v}\right) \stackrel{\text { def }}{=} \operatorname{det}\left(\begin{array}{ccccc}
c_{1} & c_{2} & \cdots & c_{m-1} & c_{m+1} \\
c_{2} & c_{3} & \cdots & c_{m} & c_{m+2} \\
\vdots & \vdots & \ddots & \vdots & \vdots \\
c_{m} & c_{m+1} & \cdots & c_{2 m-2} & c_{2 m}
\end{array}\right) .
\end{gathered}
$$

Remark 1. The matrix for $\chi_{m}$ is obtained from the matrix for $H_{m+1}^{(1)}$ by deleting the last row and next to last column [14,20,25]. For $n=1, H_{1}^{(1)}=c_{1}$ and $\chi_{1}=c_{2}$. Determinants $H_{m}^{(n)}$ and $\chi_{m}$ are named as persymmetric determinants (or) Turanian determinants (or) Hankel determinants.

The relation between FPS and Regular C fraction is given as lemma [14], (Theorem 7.2, pp 223-226, [20]), [25].

Lemma 1. When the Regular C fraction converges to FPS.

$$
1+\sum_{v=1}^{\infty} c_{v} z^{v}=1+\coprod_{n=1}^{\infty} \frac{a_{n} u}{1} ;\left(a_{n} \neq 0\right)
$$

then,

$$
\begin{gathered}
H_{m}^{(1)}\left(\left[c_{v}\right]\right) \neq 0 \quad, \quad H_{m}^{(2)}\left(\left[c_{v}\right]\right) \neq 0 \quad \text { and } \quad a_{1}=H_{1}^{(1)}\left(\left[c_{v}\right]\right) ;(m \geq 1) . \\
a_{2 m}=-\frac{H_{m-1}^{(1)} H_{m}^{(2)}}{H_{m}^{(1)} H_{m-1}^{(2)}} \text { and } a_{2 m+1}=-\frac{H_{m+1}^{(1)} H_{m-1}^{(2)}}{H_{m}^{(1)} H_{m}^{(2)}} ;(m \geq 1) .
\end{gathered}
$$

where $H_{0}^{(1)}=H_{0}^{(2)}=1$. Conversely if Eqs (6) and (7) holds then Eq (5) holds true. Also,

$$
H_{m}^{(2)}\left(\left[c_{v}\right]\right)=(-1)^{m} H_{m}^{(1)}\left(\left[c_{v}\right]\right) \prod_{j=1}^{m} a_{2 j}=(-1)^{m} H_{m+1}^{(1)}\left(\left[c_{v}\right]\right) \prod_{j=1}^{m} \frac{1}{a_{2 j+1}} ;(m \geq 1)
$$

\section{Main results 1 : Sumudu transform of Dixon elliptic functions $(\alpha \neq 0)$}

Laplace transform of DEF $\operatorname{sm}(x, \alpha), \operatorname{cm}(x, \alpha)$ and $\operatorname{sm}(x, \alpha)(\operatorname{cm}(x, \alpha))$ given as QCF in [14]. In this work Sumudu transform Eq (1) of DEF $\operatorname{sm}^{N}(x, \alpha) ; N \geq 1, \operatorname{sm}^{N}(x, \alpha) c m(x, \alpha) ; N \geq 0$ and $\operatorname{sm}^{N}(x, \alpha) \mathrm{cm}^{2}(x, \alpha) ; N \geq 0$ for arbitrary powers derived as QCF. Followed by assuming the denominator of QCF be 1, using Lemma 1, Hankel determinants are calculated. The following three theorems are main results of this work. 
Theorem 1. Sumudu transform of $\operatorname{DEF} \operatorname{sm}^{N}(x, \alpha) ; N \geq 1$ as QCF given by the following enumerates:

(i) For $j \geq 1$.

$$
\mathbb{S}[\operatorname{sm}(x, \alpha)]=\frac{u}{(1-2 \alpha u)(1+\alpha u)+} \mathbf{K}_{n=2}^{\infty} \frac{a_{n} u^{3}}{b_{n}(u)}\left\{\begin{array}{l}
a_{2 j}=(3 j-2)(3 j-1)^{2} \\
b_{2 j}(u)=(1-(6 j-1) \alpha u) \\
a_{2 j+1}=(3 j)^{2}(3 j+1) \\
b_{2 j+1}(u)=(1-2(3 j+1) \alpha u)(1+(3 j+1) \alpha u)
\end{array}\right.
$$

(ii) For $j \geq 1$.

$$
\begin{aligned}
\mathbb{S}\left[\operatorname{sm}^{2}(x, \alpha)\right]= & \frac{1}{(1-\alpha u)+} \mathbf{K}_{n=2}^{\infty} \frac{a_{n} u^{3}}{b_{n}(u)}\left\{\begin{array}{l}
a_{2 j}=(3 j-2)^{2}(3 j-1) \\
b_{2 j}(u)=(1-2(3 j-1) \alpha u)(1+(3 j-1) \alpha u) \\
a_{2 j+1}=(3 j-1)(3 j)^{2} \\
b_{2 j+1}(u)=(1-(6 j+1) \alpha u)
\end{array}\right. \\
& \times \frac{2 u^{2}}{(1-4 \alpha u)(1+2 \alpha u)+} \mathbf{K}_{n=2}^{\infty} \frac{a_{n} u^{3}}{b_{n}(u)}\left\{\begin{array}{l}
a_{2 j}=(3 j-1)(3 j)^{2} \\
b_{2 j}(u)=(1-(6 j+1) \alpha u) \\
a_{2 j+1}=(3 j+1)^{2}(3 j+2) \\
b_{2 j+1}(u)=(1-2(3 j+2) \alpha u)(1+(3 j+2) \alpha u)
\end{array}\right.
\end{aligned}
$$

(iii) Let $N=3,6,9,12, \cdots$ and $j \geq 1$.

$$
\begin{aligned}
\mathbb{S}\left[\operatorname{sm}^{N}(x, \alpha)\right]= & \prod_{i=1}^{\frac{N}{3}} \frac{(3 i-2) u}{(1-(6 i-3) \alpha u)+} \mathbf{K}_{n=2}^{\infty} \frac{a_{n} u^{3}}{b_{n}(u)}\left\{\begin{array}{l}
a_{2 j}=(3 j+3 i-4)^{2}(3 j+3 i-3) \\
b_{2 j}(u)=(1-2(3 j+3 i-3) \alpha u)(1+(3 j+3 i-3) \alpha u) \\
a_{2 j+1}=(3 j+3 i-3)(3 j+3 i-2)^{2} \\
b_{2 j+1}(u)=(1-(6 j+6 i-3) \alpha u)
\end{array}\right. \\
& \times \prod_{i=1}^{\frac{N}{3}} \frac{(3 i-1)(3 i) u^{2}}{(1-2(3 i) \alpha u)(1+(3 i) \alpha u)+} \mathbf{K}_{n=2}^{\infty} \frac{a_{n} u^{3}}{b_{n}(u)}\left\{\begin{array}{l}
a_{2 j}=(3 j+3 i-3)(3 j+3 i-2)^{2} \\
b_{2 j}(u)=(1-(6 j+6 i-3) \alpha u) \\
a_{2 j+1}=(3 j+3 i-1)^{2}(3 j+3 i) \\
b_{2 j+1}(u)=(1-2(3 j+3 i) \alpha u)(1+(3 j+3 i) \alpha u)
\end{array}\right.
\end{aligned}
$$

(iv) Let $N=4,7,10,13, \cdots$ and $j \geq 1$.

$$
\begin{aligned}
& \mathbb{S}\left[\operatorname{sm}^{N}(x, \alpha)\right]=\frac{u}{(1-2 \alpha u)(1+\alpha u)+} \mathbf{K}_{n=2}^{\infty} \frac{a_{n} u^{3}}{b_{n}(u)}\left\{\begin{array}{l}
a_{2 j}=(3 j-2)(3 j-1)^{2} \\
b_{2 j}(u)=(1-(6 j-1) \alpha u) \\
a_{2 j+1}=(3 j)^{2}(3 j+1) \\
b_{2 j+1}(u)=(1-2(3 j+1) \alpha u)(1+(3 j+1) \alpha u)
\end{array}\right. \\
& \times \prod_{i=1}^{\frac{N-1}{3}} \frac{(3 i-1) u}{(1-(6 i-1) \alpha u)+} \mathbf{K}_{n=2}^{\infty} \frac{a_{n} u^{3}}{b_{n}(u)}\left\{\begin{array}{l}
a_{2 j}=(3 j+3 i-3)^{2}(3 j+3 i-2) \\
b_{2 j}(u)=(1-2(3 j+3 i-2) \alpha u)(1+(3 j+3 i-2) \alpha u) \\
a_{2 j+1}=(3 j+3 i-2)(3 j+3 i-1)^{2} \\
b_{2 j+1}(u)=(1-(6 j+6 i-1) \alpha u)
\end{array}\right. \\
& \times \prod_{i=1}^{\frac{N-1}{3}} \frac{(3 i)(3 i+1) u^{2}}{(1-2(3 i+1) \alpha u)(1+(3 i+1) \alpha u)+} \mathbf{K}_{n=2}^{\infty} \frac{a_{n} u^{3}}{b_{n}(u)}\left\{\begin{array}{l}
a_{2 j}=(3 j+3 i-2)(3 j+3 i-1)^{2} \\
b_{2 j}(u)=(1-(6 j+6 i-1) \alpha u) \\
a_{2 j+1}=(3 j+3 i)^{2}(3 j+3 i+1) \\
b_{2 j+1}(u)=(1-2(3 j+3 i+1) \alpha u)(1+(3 j+3 i+1) \alpha u)
\end{array}\right.
\end{aligned}
$$


(v) Let $N=5,8,11,14, \cdots$ and $j \geq 1$.

$$
\begin{aligned}
& \mathbb{S}\left[\operatorname{sm}^{N}(x, \alpha)\right]=\frac{1}{(1-\alpha u)+} \mathbf{K}_{n=2}^{\infty} \frac{a_{n} u^{3}}{b_{n}(u)}\left\{\begin{array}{l}
a_{2 j}=(3 j-2)^{2}(3 j-1) \\
b_{2 j}(u)=(1-2(3 j-1) \alpha u)(1+(3 j-1) \alpha u) \\
a_{2 j+1}=(3 j-1)(3 j)^{2} \\
b_{2 j+1}(u)=(1-(6 j+1) \alpha u)
\end{array}\right. \\
& \times \frac{2 u^{2}}{(1-4 \alpha u)(1+2 \alpha u)+} \mathbf{K}_{n=2}^{\infty} \frac{a_{n} u^{3}}{b_{n}(u)}\left\{\begin{array}{l}
a_{2 j}=(3 j-1)(3 j)^{2} \\
b_{2 j}(u)=(1-(6 j+1) \alpha u) \\
a_{2 j+1}=(3 j+1)^{2}(3 j+2) \\
b_{2 j+1}(u)=(1-2(3 j+2) \alpha u)(1+(3 j+2) \alpha u)
\end{array}\right. \\
& \times \prod_{i=1}^{\frac{N-2}{3}} \frac{(3 i) u}{(1-(6 i+1) \alpha u)+} \mathbf{K}_{n=2}^{\infty} \frac{a_{n} u^{3}}{b_{n}(u)}\left\{\begin{array}{l}
a_{2 j}=(3 j+3 i-2)^{2}(3 j+3 i-1) \\
b_{2 j}(u)=(1-2(3 j+3 i-1) \alpha u)(1+(3 j+3 i-1) \alpha u) \\
a_{2 j+1}=(3 j+3 i-1)(3 j+3 i)^{2} \\
b_{2 j+1}(u)=(1-(6 j+6 i+1) \alpha u)
\end{array}\right. \\
& \times \prod_{i=1}^{\frac{N-2}{3}} \frac{(3 i+1)(3 i+2) u^{2}}{(1-2(3 i+2) \alpha u)(1+(3 i+2) \alpha u)+} \mathbf{K}_{n=2}^{\infty} \frac{a_{n} u^{3}}{b_{n}(u)}\left\{\begin{array}{l}
a_{2 j}=(3 j+3 i-1)(3 j+3 i)^{2} \\
b_{2 j}(u)=(1-(6 j+6 i+1) \alpha u) \\
a_{2 j+1}=(3 j+3 i+1)^{2}(3 j+3 i+2) \\
b_{2 j+1}(u)=(1-2(3 j+3 i+2) \alpha u)(1+(3 j+3 i+2) \alpha u)
\end{array}\right.
\end{aligned}
$$

Proof. Defining the Sumudu transform of DEF by integral equations, Let $N=0,1,2, \cdots$.

$$
\begin{gathered}
\mathbb{S}\left[\operatorname{sm}^{N}(x, \alpha)\right]=A_{N}=\int_{0}^{\infty} e^{-x} \operatorname{sm}^{N}(x u, \alpha) d x . \\
\mathbb{S}\left[\operatorname{sm}^{N}(x, \alpha) c m(x, \alpha)\right]=B_{N}=\int_{0}^{\infty} e^{-x} \operatorname{sm}^{N}(x u, \alpha) c m(x u, \alpha) d x . \\
\mathbb{S}\left[\operatorname{sm}^{N}(x, \alpha) \mathrm{cm}^{2}(x, \alpha)\right]=C_{N}=\int_{0}^{\infty} e^{-x} \operatorname{sm}^{N}(x u, \alpha) c m^{2}(x u, \alpha) d x .
\end{gathered}
$$

By parts method, using Eqs (2) - (4), with $A_{0}=1$ leads to the following:

$$
\begin{aligned}
& A_{1}=u C_{0}-\alpha u A_{1} . \\
& A_{2}=2 u C_{1}-2 \alpha u A_{2} . \\
& A_{3}=3 u C_{2}-3 \alpha u A_{3} . \\
& A_{N}=N u C_{N-1}-N \alpha u A_{N} .
\end{aligned}
$$

Solving with the recurrences of Eqs (15) and (16) yields the following QCF:

$$
\begin{aligned}
\frac{A_{N}}{B_{N-2}} & =\frac{(N-1) N u^{2}}{(1-2 N \alpha u)(1+N \alpha u)+N(N+1) u^{2} \frac{B_{N+1}}{A_{N}}} ;(N \geq 2) . \\
\frac{B_{N}}{A_{N-1}} & =\frac{N u}{(1-(2 N+1) \alpha u)+(N+1) u \frac{A_{N+2}}{B_{N}}} ;(N \geq 2) .
\end{aligned}
$$


When $N=1,2$ and 3 .

$$
\begin{aligned}
& A_{1}=\frac{u}{(1-2 \alpha u)(1+\alpha u)+2 u^{2} \frac{B_{2}}{A_{1}}} . \\
& A_{2}=B_{0} \times \frac{2 u^{2}}{(1-4 \alpha u)(1+2 \alpha u)+6 u^{2} \frac{B_{3}}{A_{2}}} . \\
& A_{3}=B_{1} \times \frac{6 u^{2}}{(1-6 \alpha u)(1+3 \alpha u)+12 u^{2} \frac{B_{4}}{A_{3}}} .
\end{aligned}
$$

Now Eq (9) obtained from Eq (19) by iterating with Eqs (17) and (18). Next Eq (10) obtained from Eq (20) by iterating with Eqs (17) and (18) where $B_{0}$ is derived from Eq (15). Following the same procedure Eqs (11) - (14) derived upon continuous iteration of Eqs (17) and (18) and after the mathematical simplifications.

Theorem 2. Sumudu transform of $s m^{N}(x, \alpha) c m(x, \alpha) ; N \geq 0$ as QCF is given by following equations:

(i) For $j \geq 1$.

$$
\mathbb{S}[c m(x, \alpha)]=\frac{1}{(1-\alpha u)+} \mathbf{K}_{n=2}^{\infty} \frac{a_{n} u^{3}}{b_{n}(u)}\left\{\begin{array}{l}
a_{2 j}=(3 j-2)^{2}(3 j-1) \\
b_{2 j}(u)=(1-2(3 j-1) \alpha u)(1+(3 j-1) \alpha u) \\
a_{2 j+1}=(3 j-1)(3 j)^{2} \\
b_{2 j+1}(u)=(1-(6 j+1) \alpha u)
\end{array}\right.
$$

(ii) For $j \geq 1$.

$$
\mathbb{S}[\operatorname{sm}(x, \alpha) c m(x, \alpha)]=\frac{u}{(1-3 \alpha u)+} \mathbf{K}_{n=2}^{\infty} \frac{a_{n} u^{3}}{b_{n}(u)}\left\{\begin{array}{l}
a_{2 j}=(3 j-1)^{2}(3 j) \\
b_{2 j}(u)=(1-2(3 j) \alpha u)(1+(3 j) \alpha u) \\
a_{2 j+1}=(3 j)(3 j+1)^{2} \\
b_{2 j+1}(u)=(1-(6 j+3) \alpha u)
\end{array}\right.
$$

(iii) For $j \geq 1$.

$$
\begin{aligned}
\mathbb{S}\left[\operatorname{sm}^{2}(x, \alpha) c m(x, \alpha)\right]= & \frac{u}{(1-2 \alpha u)(1+\alpha u)+} \mathbf{K}_{n=2}^{\infty} \frac{a_{n} u^{3}}{b_{n}(u)}\left\{\begin{array}{l}
a_{2 j}=(3 j-2)(3 j-1)^{2} \\
b_{2 j}(u)=(1-(6 j-1) \alpha u) \\
a_{2 j+1}=(3 j)^{2}(3 j+1) \\
b_{2 j+1}(u)=(1-2(3 j+1) \alpha u)(1+(3 j+1) \alpha u)
\end{array}\right. \\
& \times \frac{2 u}{(1-5 \alpha u)+} \mathbf{K}_{n=2}^{\infty} \frac{a_{n} u^{3}}{b_{n}(u)}\left\{\begin{array}{l}
a_{2 j}=(3 j)^{2}(3 j+1) \\
b_{2 j}(u)=(1-2(3 j+1) \alpha u)(1+(3 j+1) \alpha u) \\
a_{2 j+1}=(3 j+1)(3 j+2)^{2} \\
b_{2 j+1}(u)=(1-(6 j+5) \alpha u)
\end{array}\right.
\end{aligned}
$$


(iv) Let $N=3,6,9,12, \cdots$ and $j \geq 1$.

$$
\begin{aligned}
& \mathbb{S}\left[s m^{N}(x, \alpha) c m(x, \alpha)\right]=\frac{1}{(1-\alpha u)+} \mathbf{K}_{n=2}^{\infty} \frac{a_{n} u^{3}}{b_{n}(u)}\left\{\begin{array}{l}
a_{2 j}=(3 j-2)^{2}(3 j-1) \\
b_{2 j}(u)=(1-2(3 j-1) \alpha u)(1+(3 j-1) \alpha u) \\
a_{2 j+1}=(3 j-1)(3 j)^{2} \\
b_{2 j+1}(u)=(1-(6 j+1) \alpha u)
\end{array}\right. \\
& \times \prod_{i=1}^{\frac{N}{3}} \frac{(3 i-2)(3 i-1) u^{2}}{(1-2(3 i-1) \alpha u)(1+(3 i-1) \alpha u)+} \mathbf{K}_{n=2}^{\infty} \frac{a_{n} u^{3}}{b_{n}(u)}\left\{\begin{array}{l}
a_{2 j}=(3 j+3 i-4)(3 j+3 i-3)^{2} \\
b_{2 j}(u)=(1-(6 j+6 i-5) \alpha u) \\
a_{2 j+1}=(3 j+3 i-2)^{2}(3 j+3 i-1) \\
b_{2 j+1}(u)=(1-2(3 j+3 i-1) \alpha u)(1+(3 j+3 i-1) \alpha u)
\end{array}\right. \\
& \times \prod_{i=1}^{\frac{N}{3}} \frac{(3 i) u}{(1-(6 i+1) \alpha u)+} \mathbf{K}_{n=2}^{\infty} \frac{a_{n} u^{3}}{b_{n}(u)}\left\{\begin{array}{l}
a_{2 j}=(3 j+3 i-2)^{2}(3 j+3 i-1) \\
b_{2 j}(u)=(1-2(3 j+3 i-1) \alpha u)(1+(3 j+3 i-1) \alpha u) \\
a_{2 j+1}=(3 j+3 i-1)(3 j+3 i)^{2} \\
b_{2 j+1}(u)=(1-(6 j+6 i+1) \alpha u)
\end{array}\right.
\end{aligned}
$$

(v) Let $N=4,7,10,13, \cdots$ and $j \geq 1$.

$$
\begin{aligned}
& \mathbb{S}\left[\operatorname{sm}^{N}(x, \alpha) c m(x, \alpha)\right]=\frac{u}{(1-3 \alpha u)+} \mathbf{K}_{n=2}^{\infty} \frac{a_{n} u^{3}}{b_{n}(u)}\left\{\begin{array}{l}
a_{2 j}=(3 j-1)^{2}(3 j) \\
b_{2 j}(u)=(1-2(3 j) \alpha u)(1+(3 j) \alpha u) \\
a_{2 j+1}=(3 j)(3 j+1)^{2} \\
b_{2 j+1}(u)=(1-(6 j+3) \alpha u)
\end{array}\right. \\
& \times \prod_{i=1}^{\frac{N-1}{3}} \frac{(3 i-1)(3 i) u^{2}}{(1-2(3 i) \alpha u)(1+(3 i) \alpha u)+} \mathbf{K}_{n=2}^{\infty} \frac{a_{n} u^{3}}{b_{n}(u)}\left\{\begin{array}{l}
a_{2 j}=(3 j+3 i-3)(3 j+3 i-2)^{2} \\
b_{2 j}(u)=(1-(6 j+6 i-3) \alpha u) \\
a_{2 j+1}=(3 j+3 i-1)^{2}(3 j+3 i) \\
b_{2 j+1}(u)=(1-2(3 j+3 i) \alpha u)(1+(3 j+3 i) \alpha u)
\end{array}\right. \\
& \times \prod_{i=1}^{\frac{N-1}{3}} \frac{(3 i+1) u}{(1-(6 i+3) \alpha u)+} \mathbf{K}_{n=2}^{\infty} \frac{a_{n} u^{3}}{b_{n}(u)}\left\{\begin{array}{l}
a_{2 j}=(3 j+3 i-1)^{2}(3 j+3 i) \\
b_{2 j}(u)=(1-2(3 j+3 i) \alpha u)(1+(3 j+3 i) \alpha u) \\
a_{2 j+1}=(3 j+3 i)(3 j+3 i+1)^{2} \\
b_{2 j+1}(u)=(1-(6 j+6 i+3) \alpha u)
\end{array}\right.
\end{aligned}
$$


(vi) Let $N=5,8,11,14, \cdots$ and $j \geq 1$.

$$
\begin{aligned}
& \mathbb{S}\left[\operatorname{sm}^{N}(x, \alpha) c m(x, \alpha)\right]=\frac{u}{(1-2 \alpha u)(1+\alpha u)+} \mathbf{K}_{n=2}^{\infty} \frac{a_{n} u^{3}}{b_{n}(u)}\left\{\begin{array}{l}
a_{2 j}=(3 j-2)(3 j-1)^{2} \\
b_{2 j}(u)=(1-(6 j-1) \alpha u) \\
a_{2 j+1}=(3 j)^{2}(3 j+1) \\
b_{2 j+1}(u)=(1-2(3 j+1) \alpha u)(1+(3 j+1) \alpha u)
\end{array}\right. \\
& \times \frac{2 u}{(1-5 \alpha u)+} \mathbf{K}_{n=2}^{\infty} \frac{a_{n} u^{3}}{b_{n}(u)}\left\{\begin{array}{l}
a_{2 j}=(3 j)^{2}(3 j+1) \\
b_{2 j}(u)=(1-2(3 j+1) \alpha u)(1+(3 j+1) \alpha u) \\
a_{2 j+1}=(3 j+1)(3 j+2)^{2} \\
b_{2 j+1}(u)=(1-(6 j+5) \alpha u)
\end{array}\right. \\
& \times \prod_{i=1}^{\frac{N-2}{3}} \frac{(3 i)(3 i+1) u^{2}}{(1-2(3 i+1) \alpha u)(1+(3 i+1) \alpha u)+} \prod_{n=2}^{\infty} \frac{a_{n} u^{3}}{b_{n}(u)}\left\{\begin{array}{l}
a_{2 j}=(3 j+3 i-2)(3 j+3 i-1)^{2} \\
b_{2 j}(u)=(1-(6 j+6 i-1) \alpha u) \\
a_{2 j+1}=(3 j+3 i)^{2}(3 j+3 i+1) \\
b_{2 j+1}(u)=(1-2(3 j+3 i+1) \alpha u)(1+(3 j+3 i+1) \alpha u)
\end{array}\right. \\
& \times \prod_{i=1}^{\frac{N-2}{3}} \frac{(3 i+2) u}{(1-(6 i+5) \alpha u)+} \mathbf{K}_{n=2}^{\infty} \frac{a_{n} u^{3}}{b_{n}(u)}\left\{\begin{array}{l}
a_{2 j}=(3 j+3 i)^{2}(3 j+3 i+1) \\
b_{2 j}(u)=(1-2(3 j+3 i+1) \alpha u)(1+(3 j+3 i+1) \alpha u) \\
a_{2 j+1}=(3 j+3 i+1)(3 j+3 i+2)^{2} \\
b_{2 j+1}(u)=(1-(6 j+6 i+5) \alpha u)
\end{array}\right.
\end{aligned}
$$

Proof. Solving the recurrences of Eq (15):

$$
\begin{aligned}
& B_{0}=1-u A_{2}+\alpha u B_{0} . \\
& B_{1}=u-2 u A_{3}+3 \alpha u B_{1} . \\
& B_{2}=2 u A_{1}-3 u A_{4}+5 \alpha u B_{2} . \\
& B_{3}=3 u A_{2}-4 u A_{5}+7 \alpha u B_{3} . \\
& B_{N}=N u A_{N-1}-(N+1) u A_{N+2}+(2 N+1) \alpha u B_{N} .
\end{aligned}
$$

For $N=0,1$ and 2 in Eq (15) after solving with recurrences of Eqs (14) and (16):

$$
\begin{aligned}
& B_{0}=\frac{1}{(1-\alpha u)+u_{\overline{B_{0}}}^{A_{0}}} . \\
& B_{1}=\frac{u}{(1-3 \alpha u)+2 u \frac{A_{3}}{B_{1}}} . \\
& B_{0}=A_{1} \times \frac{2 u}{(1-5 \alpha u)+3 u \frac{A_{4}}{B_{2}}} .
\end{aligned}
$$

Now Eq (22) derived from Eq (28) upon iterating with Eqs (17) and (18). Eq (23) derived from Eq (29) upon iterating with Eqs (17) and (18). Eq (24) derived from Eq (30) where $A_{1}$ given by Eq (19) and both are iterated with Eqs (17) and (18). Continuing in the same way Eqs (25) - (27) obtained by iterations, mathematical calculations and simplification.

Theorem 3. Sumudu transform of $\operatorname{sm}^{N}(x, \alpha) \mathrm{cm}^{2}(x, \alpha) ; N \geq 0$ as QCF given by the following equations:

(i) For $j \geq 1$.

$$
\mathbb{S}\left[\mathrm{cm}^{2}(x, \alpha)\right]=\frac{1}{(1-2 \alpha u)+} \mathbf{K}_{n=2}^{\infty} \frac{a_{n}(u) u^{3}}{b_{n}(u)}\left\{\begin{array}{l}
a_{2 j}(u)=(3 j-2)(3 j-1)^{2}(1+(3 j+1) \alpha u) \\
b_{2 j}(u)=Y_{3 j-1} \\
a_{2 j+1}(u)=(3 j)^{2}(3 j+1)(1+(3 j-2) \alpha u) \\
b_{2 j+1}(u)=(1-(6 j+2) \alpha u)
\end{array}\right.
$$


(ii) For $j \geq 1$.

$$
\begin{aligned}
\mathbb{S}\left[\operatorname{sm}(x, \alpha) c m^{2}(x, \alpha)\right]= & \frac{1}{(1-\alpha u)+} \mathbf{K}_{n=2}^{\infty} \frac{a_{n} u^{3}}{b_{n}(u)}\left\{\begin{array}{l}
a_{2 j}=(3 j-2)^{2}(3 j-1) \\
b_{2 j}(u)=(1-2(3 j-1) \alpha u)(1+(3 j-1) \alpha u) \\
a_{2 j+1}=(3 j-1)(3 j)^{2} \\
b_{2 j+1}(u)=(1-(6 j+1) \alpha u)
\end{array}\right. \\
& \times \frac{u}{(1-4 \alpha u)+} \mathbf{K}_{n=2}^{\infty} \frac{a_{n}(u) u^{3}}{b_{n}(u)}\left\{\begin{array}{l}
a_{2 j}(u)=(3 j-1)(3 j)^{2}(1+(3 j+2) \alpha u) \\
b_{2 j}(u)=Y_{3 j} \\
a_{2 j+1}(u)=(3 j+1)^{2}(3 j+2)(1+(3 j-1) \alpha u) \\
b_{2 j+1}(u)=(1-(6 j+4) \alpha u)
\end{array}\right.
\end{aligned}
$$

(iii) For $j \geq 1$.

$$
\begin{aligned}
\mathbb{S}\left[s m^{2}(x, \alpha) c m^{2}(x, \alpha)\right]= & \frac{u}{(1-3 \alpha u)+} \mathbf{K}_{n=2}^{\infty} \frac{a_{n} u^{3}}{b_{n}(u)}\left\{\begin{array}{l}
a_{2 j}=(3 j-1)^{2}(3 j) \\
b_{2 j}(u)=(1-2(3 j) \alpha u)(1+(3 j) \alpha u) \\
a_{2 j+1}=(3 j)(3 j+1)^{2} \\
b_{2 j+1}(u)=(1-(6 j+3) \alpha u)
\end{array}\right. \\
& \times \frac{2 u}{(1-6 \alpha u)+} \mathbf{K}_{n=2}^{\infty} \frac{a_{n}(u) u^{3}}{b_{n}(u)}\left\{\begin{array}{l}
a_{2 j}(u)=(3 j)(3 j+1)^{2}(1+(3 j+3) \alpha u) \\
b_{2 j}(u)=Y_{3 j+1} \\
a_{2 j+1}(u)=(3 j+2)^{2}(3 j+3)(1+(3 j) \alpha u) \\
b_{2 j+1}(u)=(1-(6 j+6) \alpha u)
\end{array}\right.
\end{aligned}
$$

(iv) For $j \geq 1$.

$$
\begin{aligned}
\mathbb{S}\left[\mathrm{sm}^{3}(x, \alpha) \mathrm{cm}^{2}(x, \alpha)\right]= & \frac{u}{(1-2 \alpha u)(1+\alpha u)+} \mathbf{K}_{n=2}^{\infty} \frac{a_{n} u^{3}}{b_{n}(u)}\left\{\begin{array}{l}
a_{2 j}=(3 j-2)(3 j-1)^{2} \\
b_{2 j}(u)=(1-(6 j-1) \alpha u) \\
a_{2 j+1}=(3 j)^{2}(3 j+1) \\
b_{2 j+1}(u)=(1-2(3 j+1) \alpha u)(1+(3 j+1) \alpha u)
\end{array}\right. \\
& \times \frac{2 u}{(1-5 \alpha u)+} \mathbf{K}_{n=2}^{\infty} \frac{a_{n} u^{3}}{b_{n}(u)}\left\{\begin{array}{l}
a_{2 j}=(3 j)^{2}(3 j+1) \\
b_{2 j}(u)=(1-2(3 j+1) \alpha u)(1+(3 j+1) \alpha u) \\
a_{2 j+1}=(3 j+1)(3 j+2)^{2} \\
b_{2 j+1}(u)=(1-(6 j+5) \alpha u)
\end{array}\right. \\
& \times \frac{3 u}{(1-8 \alpha u)+} \mathbf{K}_{n=2}^{\infty} \frac{a_{n}(u) u^{3}}{b_{n}(u)}\left\{\begin{array}{l}
a_{2 j}(u)=(3 j+1)(3 j+2)^{2}(1+(3 j+4) \alpha u) \\
b_{2 j}(u)=Y_{3 j+2} \\
a_{2 j+1}(u)=(3 j+3)^{2}(3 j+4)(1+(3 j+1) \alpha u) \\
b_{2 j+1}(u)=(1-(6 j+8) \alpha u)
\end{array}\right.
\end{aligned}
$$


(v) Let $N=4,7,10,13, \cdots$ and $j \geq 1$.

$$
\begin{aligned}
& \mathbb{S}\left[s m^{N}(x, \alpha) c m^{2}(x, \alpha)\right]=\frac{1}{(1-\alpha u)+} \mathbf{K}_{n=2}^{\infty} \frac{a_{n} u^{3}}{b_{n}(u)}\left\{\begin{array}{l}
a_{2 j}=(3 j-2)^{2}(3 j-1) \\
b_{2 j}(u)=(1-2(3 j-1) \alpha u)(1+(3 j-1) \alpha u) \\
a_{2 j+1}=(3 j-1)(3 j)^{2} \\
b_{2 j+1}(u)=(1-(6 j+1) \alpha u)
\end{array}\right. \\
& \times \prod_{i=1}^{\frac{N-1}{3}} \frac{(3 i-2)(3 i-1) u^{2}}{(1-2(3 i-1) \alpha u)(1+(3 i-1) \alpha u)+} \mathbf{K}_{n=2}^{\infty} \frac{a_{n} u^{3}}{b_{n}(u)}\left\{\begin{array}{l}
a_{2 j}=(3 j+3 i-4)(3 j+3 i-3)^{2} \\
b_{2 j}(u)=(1-(6 j+6 i-5) \alpha u) \\
a_{2 j+1}=(3 j+3 i-2)^{2}(3 j+3 i-1) \\
b_{2 j+1}(u)=(1-2(3 j+3 i-1) \alpha u)(1+(3 j+3 i-1) \alpha u)
\end{array}\right. \\
& \times \prod_{i=1}^{\frac{N-1}{3}} \frac{(3 i) u}{(1-(6 i+1) \alpha u)+} \mathbf{K}_{n=2}^{\infty} \frac{a_{n} u^{3}}{b_{n}(u)}\left\{\begin{array}{l}
a_{2 j}=(3 j+3 i-2)^{2}(3 j+3 i-1) \\
b_{2 j}(u)=(1-2(3 j+3 i-1) \alpha u)(1+(3 j+3 i-1) \alpha u) \\
a_{2 j+1}=(3 j+3 i-1)(3 j+3 i)^{2} \\
b_{2 j+1}(u)=(1-(6 j+6 i+1) \alpha u)
\end{array}\right. \\
& \times \frac{N u}{(1-(2(N+1)) \alpha u)+} \mathbf{K}_{n=2}^{\infty} \frac{a_{n}(u) u^{3}}{b_{n}(u)}\left\{\begin{array}{l}
a_{2 j}(u)=(3 j+N-2)(3 j+N-1)^{2}(1+(3 j+N+1) \alpha u) \\
b_{2 j}(u)=Y_{3 j+N-1} \\
a_{2 j+1}(u)=(3 j+N)^{2}(3 j+N+1)(1+(3 j+N-2) \alpha u) \\
b_{2 j+1}(u)=(1-(6 j+2(N+1)) \alpha u)
\end{array}\right.
\end{aligned}
$$

(vi) Let $N=5,8,11,14, \cdots$ and $j \geq 1$.

$$
\begin{aligned}
& \mathbb{S}\left[\operatorname{sm}^{N}(x, \alpha) c m^{2}(x, \alpha)\right]=\frac{u}{(1-3 \alpha u)+} \mathbf{K}_{n=2}^{\infty} \frac{a_{n} u^{3}}{b_{n}(u)}\left\{\begin{array}{l}
a_{2 j}=(3 j-1)^{2}(3 j) \\
b_{2 j}(u)=(1-2(3 j) \alpha u)(1+(3 j) \alpha u) \\
a_{2 j+1}=(3 j)(3 j+1)^{2} \\
b_{2 j+1}(u)=(1-(6 j+3) \alpha u)
\end{array}\right. \\
& \times \prod_{i=1}^{\frac{N-2}{3}} \frac{(3 i-1)(3 i) u^{2}}{(1-2(3 i) \alpha u)(1+(3 i) \alpha u)+} \mathbf{K}_{n=2}^{\infty} \frac{a_{n} u^{3}}{b_{n}(u)}\left\{\begin{array}{l}
a_{2 j}=(3 j+3 i-3)(3 j+3 i-2)^{2} \\
b_{2 j}(u)=(1-(6 j+6 i-3) \alpha u) \\
a_{2 j+1}=(3 j+3 i-1)^{2}(3 j+3 i) \\
b_{2 j+1}(u)=(1-2(3 j+3 i) \alpha u)(1+(3 j+3 i) \alpha u)
\end{array}\right. \\
& \times \prod_{i=1}^{\frac{N-2}{3}} \frac{(3 i+1) u}{(1-(6 i+3) \alpha u)+} \mathbf{K}_{n=2}^{\infty} \frac{a_{n} u^{3}}{b_{n}(u)}\left\{\begin{array}{l}
a_{2 j}=(3 j+3 i-1)^{2}(3 j+3 i) \\
b_{2 j}(u)=(1-2(3 j+3 i) \alpha u)(1+(3 j+3 i) \alpha u) \\
a_{2 j+1}=(3 j+3 i)(3 j+3 i+1)^{2} \\
b_{2 j+1}(u)=(1-(6 j+6 i+3) \alpha u)
\end{array}\right. \\
& \times \frac{N u}{(1-(2(N+1)) \alpha u)+} \mathbf{K}_{n=2}^{\infty} \frac{a_{n}(u) u^{3}}{b_{n}(u)}\left\{\begin{array}{l}
a_{2 j}(u)=(3 j+N-2)(3 j+N-1)^{2}(1+(3 j+N+1) \alpha u) \\
b_{2 j}(u)=Y_{3 j+N-1} \\
a_{2 j+1}(u)=(3 j+N)^{2}(3 j+N+1)(1+(3 j+N-2) \alpha u) \\
b_{2 j+1}(u)=(1-(6 j+2(N+1)) \alpha u)
\end{array}\right.
\end{aligned}
$$


(vii) Let $N=6,9,12,15, \cdots$ and $j \geq 1$.

$$
\begin{aligned}
& \mathbb{S}\left[\operatorname{sm}^{N}(x, \alpha) c m^{2}(x, \alpha)\right]=\frac{u}{(1-2 \alpha u)(1+\alpha u)+} \mathbf{K}_{n=2}^{\infty} \frac{a_{n} u^{3}}{b_{n}(u)}\left\{\begin{array}{l}
a_{2 j}=(3 j-2)(3 j-1)^{2} \\
b_{2 j}(u)=(1-(6 j-1) \alpha u) \\
a_{2 j+1}=(3 j)^{2}(3 j+1) \\
b_{2 j+1}(u)=(1-2(3 j+1) \alpha u)(1+(3 j+1) \alpha u)
\end{array}\right. \\
& \times \frac{2 u}{(1-5 \alpha u)+} \mathbf{K}_{n=2}^{\infty} \frac{a_{n} u^{3}}{b_{n}(u)}\left\{\begin{array}{l}
a_{2 j}=(3 j)^{2}(3 j+1) \\
b_{2 j}(u)=(1-2(3 j+1) \alpha u)(1+(3 j+1) \alpha u) \\
a_{2 j+1}=(3 j+1)(3 j+2)^{2} \\
b_{2 j+1}(u)=(1-(6 j+5) \alpha u)
\end{array}\right. \\
& \times \prod_{i=1}^{\frac{N-3}{3}} \frac{(3 i)(3 i+1) u^{2}}{(1-2(3 i+1) \alpha u)(1+(3 i+1) \alpha u)+} \mathbf{K}_{n=2}^{\infty} \frac{a_{n} u^{3}}{b_{n}(u)}\left\{\begin{array}{l}
a_{2 j}=(3 j+3 i-2)(3 j+3 i-1)^{2} \\
b_{2 j}(u)=(1-(6 j+6 i-1) \alpha u) \\
a_{2 j+1}=(3 j+3 i)^{2}(3 j+3 i+1) \\
b_{2 j+1}(u)=(1-2(3 j+3 i+1) \alpha u)(1+(3 j+3 i+1) \alpha u)
\end{array}\right. \\
& \times \prod_{i=1}^{\frac{N-3}{3}} \frac{(3 i+2) u}{(1-(6 i+5) \alpha u)+} \mathbf{K}_{n=2}^{\infty} \frac{a_{n} u^{3}}{b_{n}(u)}\left\{\begin{array}{l}
a_{2 j}=(3 j+3 i)^{2}(3 j+3 i+1) \\
b_{2 j}(u)=(1-2(3 j+3 i+1) \alpha u)(1+(3 j+3 i+1) \alpha u) \\
a_{2 j+1}=(3 j+3 i+1)(3 j+3 i+2)^{2} \\
b_{2 j+1}(u)=(1-(6 j+6 i+5) \alpha u)
\end{array}\right. \\
& \times \frac{N u}{(1-(2(N+1)) \alpha u)+} \mathbf{K}_{n=2}^{\infty} \frac{a_{n}(u) u^{3}}{b_{n}(u)}\left\{\begin{array}{l}
a_{2 j}(u)=(3 j+N-2)(3 j+N-1)^{2}(1+(3 j+N+1) \alpha u) \\
b_{2 j}(u)=Y_{3 j+N-1} \\
a_{2 j+1}(u)=(3 j+N)^{2}(3 j+N+1)(1+(3 j+N-2) \alpha u) \\
b_{2 j+1}(u)=(1-(6 j+2(N+1)) \alpha u)
\end{array}\right.
\end{aligned}
$$

Proof. Evaluating by parts Eq (16) gives:

$$
\begin{aligned}
& C_{0}=1-2 u B_{2}+2 \alpha u C_{0} . \\
& C_{1}=u B_{0}-3 u B_{3}+4 \alpha u C_{1} . \\
& C_{2}=2 u B_{1}-4 u B_{4}+6 \alpha u C_{2} . \\
& C_{3}=3 u B_{2}-5 u B_{5}+8 \alpha u C_{3} . \\
& C_{N}=N u B_{N-1}-(N+2) u B_{N+2}+(2 N+2) \alpha u C_{N} .
\end{aligned}
$$

Solving with recurrences of Eqs (14) and (15) yields the QCF:

$$
\begin{aligned}
\frac{C_{N}}{B_{N-1}} & =\frac{N u}{(1-(2 N+2) \alpha u)+(N+2) u \frac{B_{N+2}}{C_{N}}} ;(N \geq 1) . \\
\frac{B_{N}}{C_{N-2}} & =\frac{(N-1) N(1+(N+2) \alpha u) u^{2}}{Y_{N}+(N+1)(N+2)(1+(N-1) \alpha u) u^{2} \frac{C_{N+1}}{B_{N}}} ;(N \geq 2) .
\end{aligned}
$$

where,

$$
Y_{N}=Y_{N}(u, \alpha)=(1-(2 N+1) \alpha u)(1+(N-1) \alpha u)(1+(N+2) \alpha u) .
$$


For $N=0,1$ and 2 in Eq (16).

$$
\begin{aligned}
& C_{0}=\frac{1}{(1-2 \alpha u)+2 u \frac{B_{2}}{C_{0}}} . \\
& C_{1}=B_{0} \times \frac{u}{(1-4 \alpha u)+3 u \frac{B_{3}}{C_{1}}} . \\
& C_{2}=B_{1} \times \frac{2 u}{(1-6 \alpha u)+4 u \frac{B_{4}}{C_{2}}} .
\end{aligned}
$$

Hence Eq (31) obtained by iterating Eqs (38) and (39) starting with Eq (41). Eq (32) obtained from the Eq (42) with $B_{0}$ given by Eq (28) iterating both respectively with Eqs (17), (18) and Eqs (38), (39). Following the same way Eq (33) obtained from (43). Eqs (34) - (37) derived by continuous iteration of Eqs (38) and (39) after mathematical calculations and simplification yields the results.

\section{Main results 2 : Applications - Hankel determinants calculations}

By assuming the denominator of QCF to 1, QCF converted to Regular C fractions. So that Lemma 1 can be applied to QCF for deriving their Hankel determinants. In Lemma $1 H_{(.)}^{(1)}\left(\left[c_{v}\right]\right)$ represents the Hankel determinants of Associated continued fractions. As QCF is dealing in this article, Quasi Associated continued fractions are considered which are discussed in detail in [11]. Therefore $H_{(.)}^{(1)}\left(\left[c_{v}\right]\right)$ of Lemma 1 are taken from [11]. The main results of this section are given in the following theorems which are Hankel determinants of the Theorems 1 - 3 respectively.

Theorem 4. Hankel determinants corresponding to Theorem 1 are given by the following equations:

(i)

$$
\begin{aligned}
& H_{1}^{(2)}\left([\operatorname{sm}(x, \alpha)]_{3 v+1}\right)=-4(1-5 \alpha u) . \\
& H_{2}^{(2)}\left([\operatorname{sm}(x, \alpha)]_{3 v+1}\right)=2400(1-5 \alpha u)^{2} E_{4} . \\
& H_{m}^{(2)}\left([\operatorname{sm}(x, \alpha)]_{3 v+1}\right)=(-1)^{m} 6^{(m-1)}(1-5 \alpha u)^{m} \prod_{j=0}^{m-1} E_{3 j+4}^{(m-j-1)} \prod_{j=1}^{m-2} H_{3 j+1}^{(m-j-1)} \prod_{j=1}^{m}(3 j-2)(3 j-1)^{2} ; m \geq 3 .
\end{aligned}
$$

(ii)

$$
\begin{aligned}
& H_{1}^{(2)}\left(\left[\operatorname{sm}^{2}(x, \alpha)\right]_{3 v+2}\right)=-36(1-7 \alpha u) . \\
& H_{m}^{(2)}\left(\left[\operatorname{sm}^{2}(x, \alpha)\right]_{3 v+2}\right)=(-1)^{m} 2^{m}(1-7 \alpha u)^{m} \prod_{j=1}^{m-1} E_{3 j+2}^{(m-j)} H_{3 j-1}^{(m-j)} \prod_{j=1}^{m}(3 j-1)(3 j)^{2} ; m \geq 2 .
\end{aligned}
$$

(iii) Let $N=3,6,9,12, \cdots$.

$$
\begin{aligned}
& H_{1}^{(2)}\left(\left[\operatorname{sm}^{N}(x, \alpha)\right]_{3 v+N}\right)=-(3 i)(3 i+1)^{2} E_{3 i} . \\
& H_{m}^{(2)}\left(\left[\operatorname{sm}^{N}(x, \alpha)\right]_{3 v+N}\right)=(-1)^{m} E_{3 i}^{m} \prod_{j=1}^{m-1} E_{3 j+3 i}^{(m-j)} H_{3 j+3 i-3}^{(m-j)} \prod_{j=1}^{m}(3 j+3 i-3)(3 j+3 i-2)^{2} ; m \geq 2 .
\end{aligned}
$$

where $i=1,2,3, \cdots, \frac{N}{3}$. 
(iv) Let $N=4,7,10,13, \cdots$.

$$
\begin{aligned}
& H_{1}^{(2)}\left(\left[\operatorname{sm}^{N}(x, \alpha)\right]_{3 v+N}\right)=-(3 i+1)(3 i+2)^{2} E_{3 i+1} . \\
& H_{m}^{(2)}\left(\left[\operatorname{sm}^{N}(x, \alpha)\right]_{3 v+N}\right)=(-1)^{m} E_{3 i+1}^{m} \prod_{j=1}^{m-1} E_{3 j+3 i+1}^{(m-j)} H_{3 j+3 i-2}^{(m-j)} \prod_{j=1}^{m}(3 j+3 i-2)(3 j+3 i-1)^{2} ; m \geq 2 .
\end{aligned}
$$

where $i=1,2,3, \cdots, \frac{N-1}{3}$.

(v) Let $N=5,8,11,14, \cdots$.

$$
\begin{aligned}
& H_{1}^{(2)}\left(\left[\operatorname{sm}^{N}(x, \alpha)\right]_{3 v+N}\right)=-(3 i+2)(3 i+3)^{2} E_{3 i} . \\
& H_{m}^{(2)}\left(\left[\operatorname{sm}^{N}(x, \alpha)\right]_{3 v+N}\right)=(-1)^{m} E_{3 i+2}^{m} \prod_{j=1}^{m-1} E_{3 j+3 i+2}^{(m-j)} H_{3 j+3 i-1}^{(m-j)} \prod_{j=1}^{m}(3 j+3 i-1)(3 j+3 i)^{2} ; m \geq 2 .
\end{aligned}
$$

where $i=1,2,3, \cdots, \frac{N-2}{3}$.

Here $E_{(.)}$and $H_{(.)}$are the polynomials in $u$ and $\alpha$ given in [11].

$$
\begin{aligned}
& E_{N}=E_{N}(u, \alpha)=(N-2)(N-1) N(1-(2 N+3) \alpha u) ;(N \geq 3) . \\
& H_{N}=H_{N}(u, \alpha)=N(N+1)(N+2)(1-(2 N-3) \alpha u) ;(N \geq 3) .
\end{aligned}
$$

Proof. Let $\left[\operatorname{sm}^{N}(x, \alpha)\right]_{3 v+N}$ be coeffecients in Maclaurin series of $\operatorname{sm}^{N}(x, \alpha) ; N=1,2,3, \cdots$.

$$
\operatorname{sm}^{N}(x, \alpha)=\sum_{v=0}^{\infty} \frac{\left[\operatorname{sm}^{N}(x, \alpha)\right]_{3 v+N} x^{3 v+N}}{(3 v+N) !} ; N=1,2,3, \cdots .
$$

Assume denominators of Theorem 1 to 1 . Now applying Eq (8) of Lemma 1 to the coeffecients of Theorem 1 where $H_{(.)}^{(1)}$ are the Hankel determinants of quasi associated continued fraction given in [11]. Now iterating and simplifying Hankel determinants of Eqs (9) - (13) given by respective enumerates of Theorem 4.

Theorem 5. Hankel determinants corresponding to Theorem 2 given by the following equations:

(i)

$$
\begin{aligned}
& H_{1}^{(2)}\left([c m(x, \alpha)]_{3 v}\right)=-2 P_{0}^{*} . \\
& H_{2}^{(2)}\left([c m(x, \alpha)]_{3 v}\right)=960\left(P_{0}^{*}\right)^{2} P_{3} . \\
& H_{m}^{(2)}\left([c m(x, \alpha)]_{3 v}\right)=(-1)^{m} 6^{m-1}\left(P_{0}^{*}\right)^{m} P_{3}^{m-1} \prod_{j=1}^{m-2} S_{3 j}^{(m-j-1)} P_{3 j+3}^{(m-j-1)} \prod_{j=1}^{m}(3 j-2)^{2}(3 j-1) ; m \geq 3 .
\end{aligned}
$$

(ii)

$$
\begin{aligned}
& H_{1}^{(2)}\left([\operatorname{sm}(x, \alpha) \operatorname{cm}(x, \alpha)]_{3 v+1}\right)=-12 P_{1}^{*} . \\
& H_{m}^{(2)}\left([\operatorname{sm}(x, \alpha) c m(x, \alpha)]_{3 v+1}\right)=(-1)^{m}\left(P_{1}^{*}\right)^{m} \prod_{j=1}^{m-1} S_{3 j-2}^{(m-j)} P_{3 j+1}^{(m-j)} \prod_{j=1}^{m}(3 j-1)^{2}(3 j) ; m \geq 2 .
\end{aligned}
$$

(iii)

$$
\begin{aligned}
& H_{1}^{(2)}\left(\left[\operatorname{sm}^{2}(x, \alpha) c m(x, \alpha)\right]_{3 v+2}\right)=-72 P_{2}^{*} . \\
& H_{m}^{(2)}\left(\left[\operatorname{sm}^{2}(x, \alpha) c m(x, \alpha)\right]_{3 v+2}\right)=(-1)^{m} 2^{m}\left(P_{2}^{*}\right)^{m} \prod_{j=1}^{m-1} S_{3 j-1}^{(m-j)} P_{3 j+2}^{(m-j)} \prod_{j=1}^{m}(3 j)^{2}(3 j+1) ; m \geq 2 .
\end{aligned}
$$


(iv) Let $N=3,6,9,12, \cdots$.

$$
\begin{aligned}
& H_{1}^{(2)}\left(\left[\operatorname{sm}^{N}(x, \alpha) c m(x, \alpha)\right]_{3 v+N}\right)=-(3 i+1)^{2}(3 i+2) P_{3 i} \\
& H_{m}^{(2)}\left(\left[\operatorname{sm}^{N}(x, \alpha) c m(x, \alpha)\right]_{3 v+N}\right)=(-1)^{m}\left(P_{3 i}\right)^{m} \prod_{j=1}^{m-1} S_{3 j+3 i-3}^{(m-j)} P_{3 j+3 i}^{(m-j)} \prod_{j=1}^{m}(3 j+3 i-2)^{2}(3 j+3 i-1) ; m \geq 2
\end{aligned}
$$

where $i=1,2,3, \cdots \frac{N}{3}$.

(v) Let $N=4,7,10,13, \cdots$.

$$
\begin{aligned}
& H_{1}^{(2)}\left(\left[\operatorname{sm}^{N}(x, \alpha) \operatorname{cm}(x, \alpha)\right]_{3 v+N}\right)=-(3 i+2)^{2}(3 i+3) P_{3 i+1} \\
& H_{m}^{(2)}\left(\left[\operatorname{sm}^{N}(x, \alpha) \operatorname{cm}(x, \alpha)\right]_{3 v+N}\right)=(-1)^{m}\left(P_{3 i+1}\right)^{m} \prod_{j=1}^{m-1} S_{3 j+3 i-2}^{(m-j)} P_{3 j+3 i+1}^{(m-j)} \prod_{j=1}^{m}(3 j+3 i-1)^{2}(3 j+3 i) ; m \geq 2
\end{aligned}
$$

where $i=1,2,3, \cdots \frac{N-1}{3}$.

(vi) Let $N=5,8,11,14, \cdots$.

$$
\begin{aligned}
& H_{1}^{(2)}\left(\left[\operatorname{sm}^{N}(x, \alpha) c m(x, \alpha)\right]_{3 v+N}\right)=-(3 i+3)^{2}(3 i+4) P_{3 i+2} \\
& H_{m}^{(2)}\left(\left[\operatorname{sm}^{N}(x, \alpha) c m(x, \alpha)\right]_{3 v+N}\right)=(-1)^{m}\left(P_{3 i+2}\right)^{m} \prod_{j=1}^{m-1} S_{3 j+3 i-1}^{(m-j)} P_{3 j+3 i+2}^{(m-j)} \prod_{j=1}^{m}(3 j+3 i)^{2}(3 j+3 i+1) ; m \geq 2
\end{aligned}
$$

where $i=1,2,3, \cdots \frac{N-2}{3}$.

Here $P_{(.)}^{*}$ and $S_{(.)}$are polynomials in $u$ and $\alpha$ given in [11].

$$
\begin{aligned}
& P_{0}^{*}=P_{0}^{*}(u, \alpha)=(1-4 \alpha u)(1+2 \alpha u) . \\
& P_{1}^{*}=P_{1}^{*}(u, \alpha)=(1-6 \alpha u)(1+3 \alpha u) . \\
& P_{2}^{*}=P_{2}^{*}(u, \alpha)=(1-8 \alpha u)(1+4 \alpha u) . \\
& P_{N}=P_{N}(u, \alpha)=(N-2)(N-1) N(1-(2 N+4) \alpha u)(1+(N+2) \alpha u) ;(N \geq 3) . \\
& S_{N}=S_{N}(u, \alpha)=(N+1)(N+2)(N+3)(1-(2 N-2) \alpha u)(1+(N-1) \alpha u) ;(N \geq 1) .
\end{aligned}
$$

Proof. Let $\left[\operatorname{sm}^{N}(x, \alpha) \operatorname{cm}(x, \alpha)\right]_{3 v+N}$ denotes the coeffecients in Maclaurin series of $\operatorname{sm}^{N}(x, \alpha) c m(x, \alpha) ; N=0,1,2, \cdots$.

$$
\operatorname{sm}^{N}(x, \alpha) \operatorname{cm}(x, \alpha)=\sum_{v=0}^{\infty} \frac{\left[s m^{N}(x, \alpha) \operatorname{cm}(x, \alpha)\right]_{3 v+N} x^{3 v+N}}{(3 v+N) !} ; N=0,1,2, \cdots .
$$

Assume denominator of Theorem 2 to 1 . Applying coeffecients of Theorem 2 in Eq (8) of Lemma 1 where $H_{(.)}^{(1)}$ are the Hankel determinants of Quasi associated continued fraction given in [11]. Iterating and simplifying leads to the Hankel determinants of Eqs (22) - (27) by respective enumerates of Theorem 5.

Theorem 6. Hankel determinants corresponding to Theorem 3 given by the following equations: 
(i)

$$
\begin{aligned}
H_{1}^{(2)}\left(\left[\mathrm{cm}^{2}(x, \alpha)\right]_{3 v}\right)= & -4 T_{0}^{*}(1+4 \alpha u) . \\
H_{2}^{(2)}\left(\mathrm{cm}^{2}([x, \alpha)]_{3 v}\right)= & 400\left(T_{0}^{*}\right)^{2} X_{0}^{*} T_{3}(1+4 \alpha u)(1+7 \alpha u) . \\
H_{m}^{(2)}\left(\left[\mathrm{cm}^{2}(x, \alpha)\right]_{3 v}\right)= & (-1)^{m}\left(T_{0}^{*}\right)^{m}\left(X_{0}^{*}\right)^{m-1} T_{3}^{m-1} \prod_{j=1}^{m-2} X_{3 j}^{(m-j-1)} T_{3 j+3}^{(m-j-1)} \\
& \times \prod_{j=1}^{m}(3 j-2)(3 j-1)^{2}(1+(3 j+1) \alpha u) ; m \geq 3 .
\end{aligned}
$$

(ii)

$$
\begin{aligned}
H_{1}^{(2)}\left(\left[\operatorname{sm}(x, \alpha) c m^{2}(x, \alpha)\right]_{3 v+1}\right)= & -18 T_{1}^{*}(1+5 \alpha u) . \\
H_{2}^{(2)}\left(\left[\operatorname{sm}(x, \alpha) c m^{2}(x, \alpha)\right]_{3 v+1}\right)= & 3240\left(T_{1}^{*}\right)^{2} X_{1}^{*} T_{4}(1+5 \alpha u)(1+8 \alpha u) . \\
H_{m}^{(2)}\left(\left[\operatorname{sm}(x, \alpha) c m^{2}(x, \alpha)\right]_{3 v+1}\right)= & (-1)^{m}\left(T_{1}^{*}\right)^{m}\left(X_{1}^{*}\right)^{m-1} T_{4}^{m-1} \prod_{j=1}^{m-2} X_{3 j+1}^{(m-j-1)} T_{3 j+4}^{(m-j-1)} \\
& \times \prod_{j=1}^{m}(3 j-1)(3 j)^{2}(1+(3 j+2) \alpha u) ; m \geq 3 .
\end{aligned}
$$

(iii)

$$
\begin{aligned}
H_{1}^{(2)}\left(\left[\mathrm{sm}^{2}(x, \alpha) \mathrm{cm}^{2}(x, \alpha)\right]_{3 v+1}\right)= & -48 T_{2}^{*}(1+6 \alpha u) . \\
H_{m}^{(2)}\left(\left[\mathrm{sm}^{2}(x, \alpha) \mathrm{cm}^{2}(x, \alpha)\right]_{3 v+1}\right)= & (-1)^{m}\left(T_{2}^{*}\right)^{m} \prod_{j=1}^{m-1} X_{3 j-1}^{(m-j)} T_{3 j+2}^{(m-j)} \\
& \times \prod_{j=1}^{m}(3 j)(3 j+1)^{2}(1+(3 j+3) \alpha u) ; m \geq 2 .
\end{aligned}
$$

(iv)

$$
\begin{aligned}
H_{1}^{(2)}\left(\left[\mathrm{sm}^{3}(x, \alpha) \mathrm{cm}^{2}(x, \alpha)\right]_{3 v+3}\right)= & -100 T_{3}(1+7 \alpha u) . \\
H_{m}^{(2)}\left(\left[\mathrm{sm}^{3}(x, \alpha) \mathrm{cm}^{2}(x, \alpha)\right]_{3 v+3}\right)= & (-1)^{m} T_{3}^{m} \prod_{j=1}^{m-1} X_{3 j}^{(m-j)} T_{3 j+3}^{(m-j)} \\
& \times \prod_{j=1}^{m}(3 j+1)(3 j+2)^{2}(1+(3 j+4) \alpha u) ; m \geq 2 .
\end{aligned}
$$

(v) Let $N=4,7,10,13, \cdots$.

$$
\begin{aligned}
H_{1}^{(2)}\left(\left[s m^{N}(x, \alpha) c m^{2}(x, \alpha)\right]_{3 v+N}\right)= & -(3 i+2)(3 i+3)^{2}(1+(3 i+5) \alpha u) T_{3 i+1} . \\
H_{m}^{(2)}\left(\left[s m^{N}(x, \alpha) c m^{2}(x, \alpha)\right]_{3 v+N}\right)= & (-1)^{m} T_{3 i+1}^{m} \prod_{j=1}^{m-1} X_{3 j+3 i-2}^{(m-j)} T_{3 j+3 i+1}^{(m-j)} \\
& \times \prod_{j=1}^{m}(3 j+3 i-1)(3 j+3 i)^{2}(1+(3 j+3 i+2) \alpha u) ; m \geq 2 .
\end{aligned}
$$

where $i=1,2,3, \cdots \frac{N-1}{3}$. 
(vi) Let $N=5,8,11,14, \cdots$.

$$
\begin{aligned}
H_{1}^{(2)}\left(\left[\operatorname{sm}^{N}(x, \alpha) c m^{2}(x, \alpha)\right]_{3 v+N}\right)= & -(3 i+3)(3 i+4)^{2}(1+(3 i+6) \alpha u) T_{3 i+2} . \\
H_{m}^{(2)}\left(\left[s m^{N}(x, \alpha) c m^{2}(x, \alpha)\right]_{3 v+N}\right)= & (-1)^{m} T_{3 i+2}^{m} \prod_{j=1}^{m-1} X_{3 j+3 i-1}^{(m-j)} T_{3 j+3 i+2}^{(m-j)} \\
& \times \prod_{j=1}^{m}(3 j+3 i)(3 j+3 i+1)^{2}(1+(3 j+3 i+3) \alpha u) ; m \geq 2 .
\end{aligned}
$$

where $i=1,2,3, \cdots \frac{N-2}{3}$.

(vii) Let $N=6,9,12,15, \cdots$.

$$
\begin{aligned}
H_{1}^{(2)}\left(\left[\mathrm{sm}^{N}(x, \alpha) c m^{2}(x, \alpha)\right]_{3 v+N}\right)= & -(3 i+4)(3 i+5)^{2}(1+(3 i+7) \alpha u) T_{3 i+3} . \\
H_{m}^{(2)}\left(\left[s m^{N}(x, \alpha) c m^{2}(x, \alpha)\right]_{3 v+N}\right)= & (-1)^{m} T_{3 i+3}^{m} \prod_{j=1}^{m-1} X_{3 j+3 i}^{(m-j)} T_{3 j+3 i+3}^{(m-j)} \\
& \times \prod_{j=1}^{m}(3 j+3 i+1)(3 j+3 i+2)^{2}(1+(3 j+3 i+4) \alpha u) ; m \geq 2 .
\end{aligned}
$$

where $i=1,2,3, \cdots \frac{N-3}{3}$.

Here $T_{(.)}^{*}, X_{(.)}^{*}, T_{(.)}$and $X_{(.)}$are the polynomials given in [11].

$$
\begin{aligned}
& T_{0}^{*}=T_{0}^{*}(u, \alpha)=(1+\alpha u)(1+4 \alpha u)(1-5 \alpha u) \\
& X_{0}^{*}=X_{0}^{*}(u, \alpha)=24(1+\alpha u) \\
& T_{1}^{*}=T_{1}^{*}(u, \alpha)=(1+2 \alpha u)(1-7 \alpha u)(1+5 \alpha u) \\
& X_{1}^{*}=X_{1}^{*}(u, \alpha)=60(1-\alpha u)(1+2 \alpha u) \\
& T_{2}^{*}=T_{2}^{*}(u, \alpha)=2(1+3 \alpha u)(1-9 \alpha u)(1+6 \alpha u) \\
& T_{N}=T_{N}(u, \alpha)=(N-2)(N-1) N(1+(N+1) \alpha u)(1+(N+4) \alpha u)(1-(2 N+5) \alpha u) ;(N \geq 3) \\
& X_{N}=X_{N}(u, \alpha)=(N+2)(N+3)(N+4)(1+(N+1) \alpha u)(1+(N-2) \alpha u)(1-(2 N-1) \alpha u) ;(N \geq 1)
\end{aligned}
$$

Proof. Let $\left[\operatorname{sm}^{N}(x, \alpha) \mathrm{cm}^{2}(x, \alpha)\right]_{3 v+N}$ be the coeffecients in Maclaurin series of $\operatorname{sm}^{N}(x, \alpha) \mathrm{cm}^{2}(x, \alpha) ; N=0,1,2, \cdots$.

$$
s m^{N}(x, \alpha) \mathrm{cm}^{2}(x, \alpha)=\sum_{v=0}^{\infty} \frac{\left[s m^{N}(x, \alpha) c m^{2}(x, \alpha)\right]_{3 v+N} x^{3 v+N}}{(3 v+N) !} ; N=0,1,2, \cdots
$$

Assume denominator of Theorem 3 to 1. Next applying the coeffecients of Theorem 3 in Eq (8) of Lemma 1 where $H_{(.)}^{(1)}$ are the Hankel determinants of Quasi associated continued fraction given in [11]. Now iterating and simplifying Hankel determinants of Eqs (31) - (37) given by respective enumerates of Theorem 6.

\section{Results and discussions}

Multiplying Eq (9) with $u$ gives the Laplace transform of $\operatorname{sm}(x, \alpha)$ in [14] (Theorem 19. page 61, [14]). Multiplication of $u$ to Eqs (22) and (23) gives the results given in [14] (Theorems 20 and 21 respectively, pp 62-63, [14]). Remaining results in Theorems 1, 2 and 3 appears in this work are new to the literature reviewed.

Letting $\alpha=0$ in Eqs (44) - (46) gives the results in [14] $\left(H_{m}^{(2)}(\right.$.$) in Theorem 16, pp 57-58, [14]). When \alpha=0$ in Eqs (55) - (57) and Eqs (58) and (59) gives the results in [14] $\left(H_{m}^{(2)}(\right.$.$) of Theorems 17$ and 18, pp 58-59, [14]) respectively. Remaining results of Theorems 4, 5 and 6 appears for first time in this work as far as the literature reviewed. 


\section{Conclusion}

In this research work we applied the Sumudu integral transform to non-zero modulus Dixon elliptic functions to derive general three term recurrences from which Quasi $C$ fractions expanded. Next by assuming the functions in the denominator of QCF we calculated the Hankel determinants $H_{m}^{2}$ of non-zero DEF without expanding their Maclaurin's series by using Lemma 1 in which Hankel determinants $H_{m}^{1}$ are used from authors previous work [11]. Results and discussions section ensures our asumptions are correct and gives the previous results. It remains the open query of Sumudu transform of $\mathrm{cm}^{3}(x, \alpha)$ and other higher powers as they lead to four term recurrences. Secondly if the assumption of denominators to 1 is restricted for the Hankel determinants of QCF is another open query which will be the further study from this work.

\section{References}

[1] O. S. Adams, Elliptic functions applied to conformal world maps, U. S. Coast and Geodetic survey special publications, No. 112, Goverment printing office, Washington, 1925.

[2] W. Al-Salam and L. Carlitz, Some determinants of Bernoulli, Euler, and related numbers. Portugal. Math., series 2, Vol-18, pp 91-99, 1959.

[3] A. Atangana and A. Kilicman, The Use of Sumudu Transform for Solving Certain Nonlinear Fractional Heat-Like Equations. Abstract and Applied Mathematics, Volume 2013, Article ID 737481, pp 1-12, 2013. http://dx.doi.org/10.1155/2013/737481.

[4] R. Bacher and P. Flajolet, Pseudo-factorials, elliptic functions, and continued fractions, The Ramanujan journal., 2009.

[5] J. Singh, D. Kumar and A. Kilicman, Homotopy Perturbation Method for Fractional Gas Dynamics Equation Using Sumudu Transform, Abstract and Applied Analysis, Volume 2013, Article ID 934060, pp 1-8, 2013. http://dx.doi.org/10.1155/2013/934060

[6] F. B. M. Belgacem and R. Silambarasan, A distinctive Sumudu treatment of trigonometric functions. Journal of Computational and Applied Mathematics, 312, pp 74-81, 2017.

[7] F. B. M. Belgacem, E. H. A. Shemas and R. Silambarasan, Sumudu computation of the transient magnetic field in a lossy medium. Applied Mathematics and Information Sciences, 11(1), pp 209-217, 2017.

[8] F. B. M. Belgacem and R. Silambarasan, Further distinctive investigations of the Sumudu transform. ICNPAA Conference, 1798, pp 020025-1-020025-9, 2017.

[9] F. B. M. Belgacem and R. Silambarasan, Sumudu transform of Dumont bimodular Jacobi elliptic functions for arbitrary powers. ICNPAA Conference, 1798, pp 020026-1-020026-9, 2017.

[10] H. Eltayeb and A. Kilicman, Application of Sumudu Decomposition Method to Solve Nonlinear System of Partial Differential Equations, Abstract and Applied Analysis, Volume 2012, Article ID 412948, pp 1-13, 2012.

[11] A. Kilicman, R. Silambarasan and O. Altun, Quasi associated continued fractions and Hankel determinants of Dixon elliptic functions via Sumudu transform. Journal of Nonlinear Sciences and Application, No. 10, pp 40004014, 2017.

[12] A. Kilicman, H. Eltayeb and R. P. Agarwal, On Sumudu Transform and System of Differential Equations, Abstract and Applied Analysis, Volume 2010, Article ID 598702, pp 1-11, 2010.

[13] L. Carlitz, Some Orthogonal Polynomials Related to Elliptic Functions. Duke Mathematical Journal, Vol-27, pp 443-459, 1960. 
[14] E. V. F. Conrad, Some continued fraction expansions of Laplace transforms of elliptic functions. PhD Thesis, The Ohio State University. 2002.

[15] E. V. F. Conrad and P. Flajolet, The Fermat cubic, elliptic functions, continued fractions, and a combinatorial excursion. Seminaire Lotharingien de Combinatoire, Vol-54 (B54g), pp 1-44, 2006.

[16] A. C. Dixon. On the doubly periodic functions arising out of the curve $x^{3}+y^{3}-3 \alpha x y=1$. The Quarterly Journal of Pure and Applied Mathematics. Vol-24, pp 167-233, 1890.

[17] A. C. Dixon, The elementary properties of the elliptic functions with examples. Macmillan anc co, London. 1894.

[18] D. Dumont, Le parametrage de la courbe d'equation $x^{3}+y^{3}=1$ (Une introduction elementaire aux fonctions elliptiques), preprint, 1988.

[19] M. Ismail and D. Masson, Some continued fractions related to elliptic functions. in: B. Berndt and F. Gesztesy, editors. Continued Fractions: from Analytic Number Theory to Constructive Approximation, (Columbia, MO, 1998). Contemporary Mathematics, Vol-236, Providence, RI, pp 149-166, 1999.

[20] W. B. Jones and W. J. Thron, Continued fractions : Analytic theory and applications. Addison-Wesley, in encyclopedia of Mathematics and its applications. 4, 1980.

[21] D. Lawden, Elliptic functions and applications. Springer-verlag, 1989.

[22] L. Lorentzen and H. Waadeland, Continued fractions with application. North-Holland. 1992.

[23] J. C. Langer and D. A. Singer, The Trefoil. Milan Journal of Mathematics, Vol-99, No. 9999, pp 1-23, 2013.

[24] T. Muir, A treatise on the theory of determinants. Dover publications, New York. 1960.

[25] S. C. Milne, Infinite families of exact sums of squares formulas, Jacobi elliptic functions, continued fractions, and Schur functions. The Ramanujan Journal, Vol-6, pp 7-149, 2002. Also published in: Developments in Mathematics, 5, Dordrecht (Kluwer, 2002).

[26] A. M. S. Mahdy, A. S. Mohamed and A. A. H. Mtawa, Implementation of the Homotopy Perturbation Sumudu Transform Method for Solving Klein-Gordon Equation, Applied Mathematics, Vol. 6, pp 617-628, 2015.

[27] M. A. Ramadan and M. S. Al-Luhaibi, Application of Sumudu Decomposition Method for Solving Linear and Nonlinear Klein-Gordon Equations, Int jour of Soft Comput Engg, Vol. 3, No. 6, pp 138-141, 2014.

[28] A. Kilicman, V. G. Gupta and B. Sharma, On the Solution of Fractional Maxwell Equations by Sumudu Transform, Journal of Mathematics Research, Vol. 2, No. 4, pp 147-151, 2010.

[29] H. Eltayeb and A. Kilicman, A Note on the Sumudu Transforms and Differential Equations, Applied Mathematical Sciences, Vol. 4, No. 22, pp 1089-1098, 2010.

[30] H.S.Wall, Note on the expansion of a power series into a continued fraction. Presented in Society, pp 97-105, 1944.

[31] H. S. Wall, Analytic theory of continued fractions. Chelsea publications. 1948. 